\title{
In Situ Growth Rate Measurement of Selective LPCVD of Tungsten
}

\author{
J. Holleman, A. Hasper, and J. Middelhoek \\ Department of Electrochemistry, University of Twente, 7500 AE Enschede, The Netherlands
}

\section{ABSTRACT}

The reflectance measurement during the selective deposition of $\mathrm{W}$ on Si covered with an insulator grating is proven to be a convenient method to monitor the $\mathrm{W}$ deposition. The reflectance change during deposition allows the in situ measurement of the deposition rate. The influence of surface roughening due to either the W growth or an etching pretreatment of the wafer is modeled, as well as the effect of selectivity loss and lateral overgrowth.

A grating has been successfully used in the past to monitor in situ the etch rate of, e.g., Si for dry trench etching (1-3).

W can be deposited selectivity by means of LPCVD using the reduction reaction of $\mathrm{WF}_{6}$ by either $\mathrm{Si}, \mathrm{H}_{2}$, or $\mathrm{SiH}_{4}(4,5)$.

The reaction conditions can be chosen so that $\mathrm{W}$ does not deposit on insulators like $\mathrm{SiO}_{2}, \mathrm{Si}_{3} \mathrm{~N}_{4}$, etc., but only grows on (semi-)conductors like $\mathrm{Si}$ and $\mathrm{Al}(4,6-8)$.

When $\mathrm{W}$ is deposited on a grating structure as presented in Fig. 1, the reflectance will change during deposition due to the changing diffraction caused by the moving $\mathrm{W}$ surface. The distance between the maximum and a minimum in reflectance is related to $\lambda / 4$, with $\lambda$ the wavelength by which the reflectance is measured.

\section{Theory}

The reflectance $(R)$ of a multilayer stack is given by

$$
R=|r|^{2}=\left(E_{1}^{-} / E_{1}^{+}\right)^{2}
$$

where $E_{1}{ }^{-}$and $E_{1}{ }^{+}$are, respectively, the amplitudes of the reflected and incident electric field vector at the first interface, $r$ is the Fresnel reflection coefficient of the multilayer stack. With more multilayer stacks side by side on the same surface the reflectance is given by

$$
R=\left|\Sigma F_{\mathrm{i}} r_{\mathrm{i}} \exp \left(j \delta_{\mathrm{i}}\right)\right|^{2}
$$

where $F_{\mathrm{i}}$ is the surface fraction covered by layer stack $\mathrm{i}, r_{\mathrm{i}}$ is the Fresnel reflection coefficient of this stack, $\delta_{i}$ is equal to $4 \pi h_{\mathrm{i}} / \lambda, h_{\mathrm{i}}$ is the height difference between the surface of layer stack $i$ and a reference level and $\lambda$ is the wavelength.

Equation [1] can be calculated using a matrix method $(9,10)$. A code was developed to calculate Eq. [2].

For the case that W is growing selectively on Si partially covered with an insulator it is convenient to take the $\mathrm{Si}$ surface level under the insulator as a reference level.

When the growing layer is suffering from roughness, which is the case for the $\mathrm{W}$ deposition $(11,12)$, the Fresnel reflectance of this stack is changing $(12,13)$. When the solid angle of observance is $<0.03 \mathrm{sr}$ the Fresnel reflectance of a rough surface is given by (13)

$$
r=\int_{-\infty}^{\infty} r(z) \cdot f(z) \exp [-4 \pi j(z / \lambda) \cos \phi] d z
$$

where $r(z)$ is the Fresnel reflection coefficient at the given angle of incidence $(\phi)$ of a smooth surface covered with $W$ thickness $z$.

The effect of the growing $W$ surface, whose roughness is increasing with $\mathrm{W}$ thickness $(11,12)$ on the total reflectance can be simulated by subdividing the $\mathrm{W}$ surface fraction in subfractions with variable thickness. The $W$ surface sub fraction $F_{\text {wab }}$ with thickness between $a$ and $b$ can be found by integration of the thickness density function $f(z)$

$$
F_{\text {wab }}=\int_{a}^{b} f(z) d z
$$

The average thickness $d_{\mathrm{F}_{\mathrm{wab}}}$ in the sub stack $F_{\text {wab }}$ is given by

$$
d_{F_{\mathrm{wab}}}=\int_{a}^{b} f(z) z d z / \int_{a}^{b} f(z) d z
$$

\section{Experimental}

Si wafers, p-type (1.0.0) $10 \Omega \mathrm{cm}$ were oxidized to $820 \mathrm{~nm}$. A $5 \mu \mathrm{m}$ grating was etched in the oxide using standard photolithography and plasma RIE etching with $\mathrm{CHF}_{3}$, followed by a $\mathrm{CF}_{4}+\mathrm{O}_{2}$ plasma etch in order to remove $20 \mathrm{~nm}$ of $\mathrm{Si}$, which is the depth of the clamage and $\mathrm{H}$ implant caused by the $\mathrm{CHF}_{3}$ plasma. After photoresist removal and prior to loading into the reactor the wafers were dipped in a $1 \% \mathrm{HF}$ solution in order to remove the native oxide on the Si. The final thickness of the oxide was $800 \mathrm{~nm}$. The grating dimensions after etching were: $\mathrm{Si}_{\mathrm{SiO}}=5.25$ : $4.75 \mu \mathrm{m}$, so in Eq. [2] $F_{\mathrm{SiO}_{2}}=0.475$ and $F_{\mathrm{Si}}=0.525$.

$\mathrm{W}$ was deposited in a single-wafer cold wall reactor with in situ reflectance measurement capabilities. A schematic drawing of the system is presented in Fig. 2. The reflectometer light source is a xenon are lamp. Both input and output fibers have a pinhole diaphragm at the end in order to keep the spot and the angle of observance small $(<0.01 \mathrm{sr})$ The photomultiplier output is fed into a computer with a phase-locked loop (PLL) amplifier on board.

$\mathrm{W}$ was deposited selectively on the Si part of the grating in a two-step process.

1. An initial $\mathrm{W}$ layer was formed by the self-limiting $\mathrm{Si}$ reduction reaction

$$
3 \mathrm{Si}+2 \mathrm{WF}_{6}=2 \mathrm{~W}+3 \mathrm{SiF}_{4}
$$

The initial layer thickness of the self-stopping Si reduction reaction as measured by the weight change of an unpatterned wafer was found to vary between $20-30 \mathrm{~nm}$. This $W$ layer consumes a Si layer which is twice the resulting $\mathrm{W}$ thickness (15). The growth rate of this initial layer is not constant but gradually becomes zero when the self-stopping thickness has been reached.

2. The initial layer was increased in thickness by either the $\mathrm{H}_{2}$ - or $\mathrm{SiH}_{4}$-reduction process

$$
\begin{gathered}
\mathrm{WF}_{6}+3 \mathrm{H}_{2}=\mathrm{W}+6 \mathrm{HF} \\
2 \mathrm{WF}_{6}+3 \mathrm{SiH}_{4}=2 \mathrm{~W}+3 \mathrm{SiF}_{4}+6 \mathrm{H}_{2}
\end{gathered}
$$

In one case the wafer was etched in situ in order to remove the native oxide with an afterglow $\mathrm{NF}_{3}$ plasma followed by the deposition of $\mathrm{W}$ via the $\mathrm{SiH}_{4}$ reduction process without deposition of the self-limiting Si-reduction process.

\section{Experimental Results, Simulations, and Discussion}

In order to simulate the reflectance relation during the initial layer formation we used the island growth model as presented elsewhere $(12,16)$. After the initial layer has been formed we assumed that further growth proceeds through layer by layer growth via either reaction [7] or [8]. The reflectance-thickness relation is calculated for $\lambda=400$

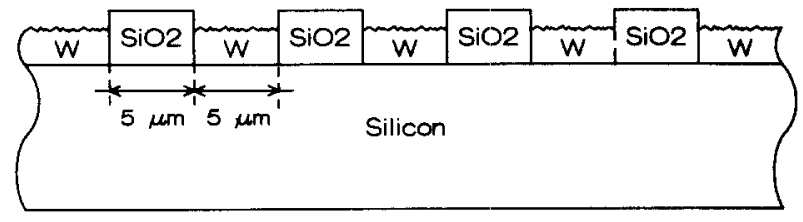

Fig. 1. Schematic drawing of the $\mathrm{SiO}_{2}$ on Si grating. 


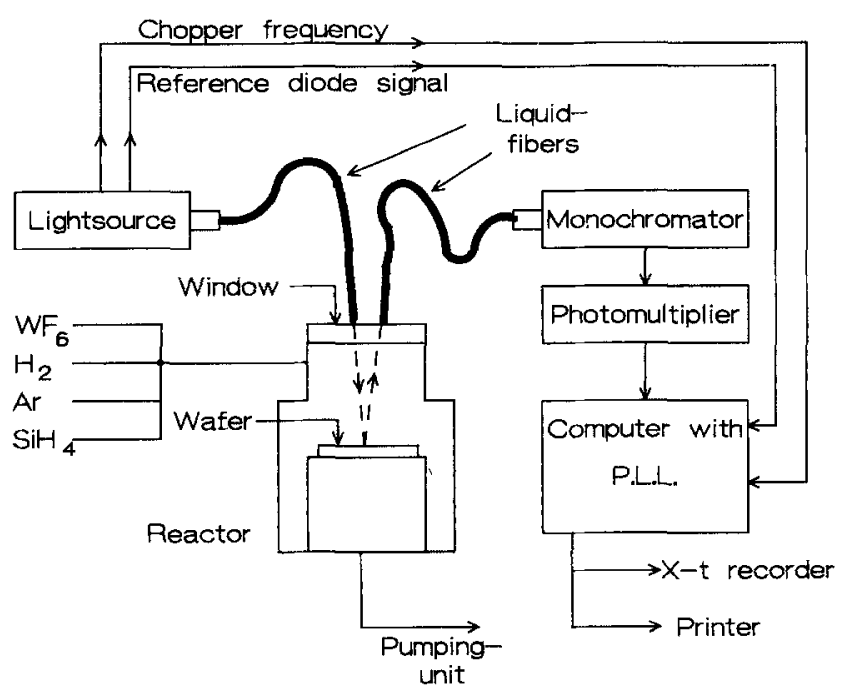

Fig. 2. Schematic drawing of the single-wafer cold-wall reactor with reflectometer setup.

$\mathrm{nm}$ and $\lambda=600 \mathrm{~nm}$ assuming an $\mathrm{rms}$ roughness $(\sigma)$ of $3 \%$ and $7 \%$ which is characteristic for the $\mathrm{SiH}_{4}$ and $\mathrm{H}_{2}$ process, respectively (12). Optical data were taken from $(17,18)$. The simulations are presented in Fig. 3 (a-d).

Note that the maximum amplitude decreases and that the minimum reflectance increases due to the increasing surface roughness. We monitored the reflectance at $\lambda=400$ and $600 \mathrm{~nm}$ during reaction [6] followed by reaction [8]; the results are presented in Fig. 4 a, b.

The reflectance is presented normalized to the reflectance at time $t=0$. The reflectance change during the Si reduction reaction is shown from $t=-60 \mathrm{~s}$ to $t=0$. The Si reduction starts after an induction period. After some time the $\mathrm{W}$ layer no longer increases in thickness because this reaction is self-limiting. The reflectance then no longer
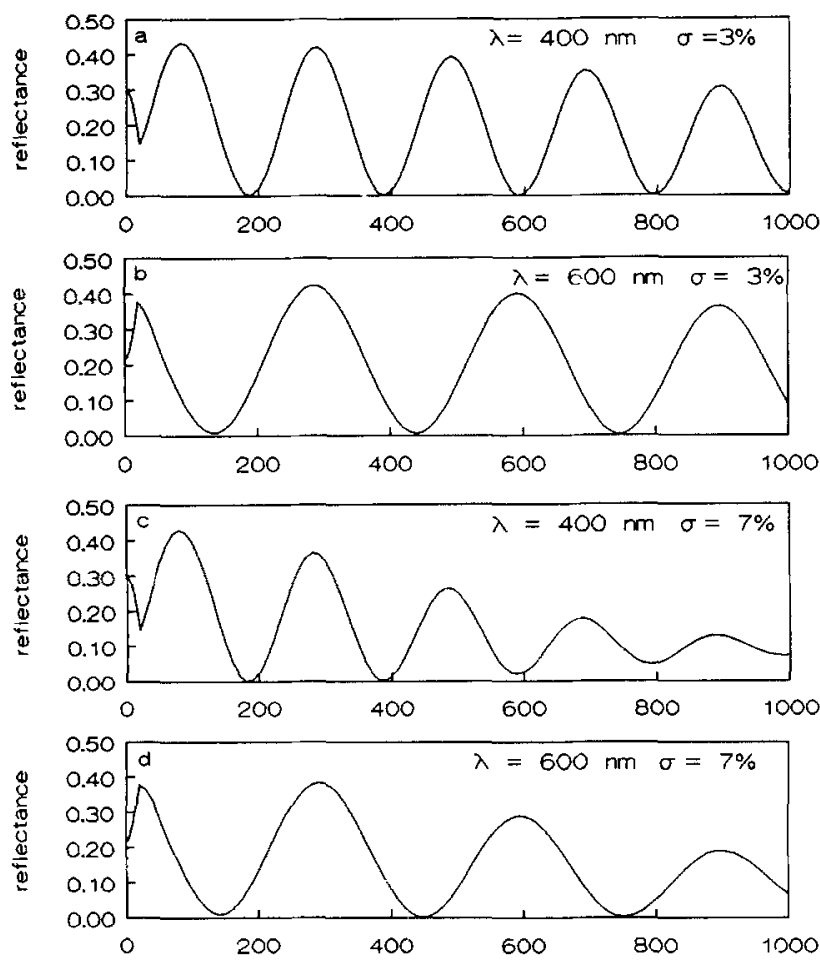

$W$ thickness $(n m)$

Fig. 3. Simulation of the reflectance as a function of the $W$ thickness on a grating with $F_{\mathrm{SiO}_{2}}=0.475$ and $F_{\mathrm{Si}}=0.525$. The oxide thickness is $800 \mathrm{~nm}$. The first $20 \mathrm{~nm}$ were assumed to be deposited via reaction [6], which includes Si consumption. The remainder was assumed to be deposited via either reaction [7] or [8].
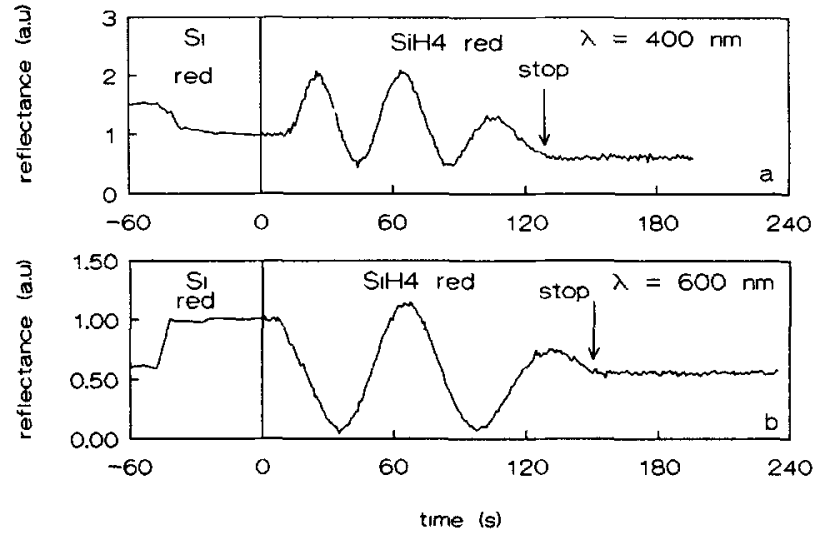

Fig. 4. Measured reflectance vs. time. The $W$ is deposited in a twostep process. The initial loyer is deposited vio reaction [6], the remainder by reaction [8].

changes. At $t=0$ the $\mathrm{SiH}_{4}$ reduction starts; again a time lag can be observed. The first induction period may be attributed to the native oxide, and indeed we found larger induction periods when the native oxide was intentionally increased in thickness by a chemical treatment. The origin of the second induction period may be the accumulation of contaminants after the W surface is formed by the Si reduction since the reactor system is a high vacuum system only. With base pressures in the $10^{-6}$ torr range one might expect a monolayer of contaminants in a time length of a few seconds. The $\mathrm{SiH}_{4}$ reduction then starts with nuclei formation which may be the source of the observed delay.

The real value in terms of reflectance can be calculated when we compare the photomultiplier output between a blank Si wafer and the specimen under study. For blank Si wafers we found at $\lambda=400 \mathrm{~nm}$ the photomultiplier output to vary between 0.75 and 0.95 . These variations may be caused by wafer bow and irreproducible susceptor positioning. We therefore prefer to present the reflectance in a.u. For comparison between calculation and measurement we calculated the value of an a.u. In Fig. $4 a$, 1 a.u. $=0.093$ on the reflectance scale. We must, however, keep in mind that based on the arguments mentioned above the accuracy is not better than $10 \%$. If we compare the results presented in Fig. 4 with the simulations presented in Fig. $3 \mathrm{a}$ and $\mathrm{b}$ we may conclude that the minimum reflectance is much higher in the $\lambda=400 \mathrm{~nm}$ case and that the amplitude becomes lower after selectivity loss was observed visually. Note that in Fig. 3 the reflectance is plotted against thickness whereas in Fig. 4 it is plotted against time.

The high minimum reflectance is probably caused by scattering. This could, for example, be the $800 \mathrm{~nm}$ oxide step which hinders higher order reflections. Multiple reflections at these steps which are not taken into account in the Kirchhoff theory may cause such deviations. Also a rough surface can cause the effects as was demonstrated in the simulations presented in Fig. 3. In order to explain the effect, an rms roughness of $50 \mathrm{~nm}$ has to be assumed as we can conclude from Fig. 5, where we calculated the maximum and minimum reflectance as a function of the rms roughness. The same roughness would not have such a serious effect in the case of a higher wavelength (compare Fig. 5a with 5b) and in fact the experimental result of the $\lambda=600 \mathrm{~nm}$ experiment does indeed not suffer that much from too high minimum reflectance. A roughness of $50 \mathrm{~nm}$ however was not observed by SEM observations after the plasma etching procedure which forms the grating in the oxide. It may however also be the case that we are dealing with a long range variation in the $\mathrm{Si}$ or $\mathrm{SiO}_{2}$ surface level.

The selectivity loss can be seen in Fig. 6 which is a SEM photograph taken from the $\lambda=400 \mathrm{~nm}$ experiment. Nearly full selectivity loss can be observed. The $\mathrm{W}$ on the $\mathrm{SiO}_{2}$ surface consists of hemispheres (more clearly visible in the insertion in Fig. 6) and the typical radius of the hemispheres can be estimated at about $250 \mathrm{~nm}$ with $\sigma$ about $100 \mathrm{~nm}$. If we assume that the radius of the hemispheres grow with 

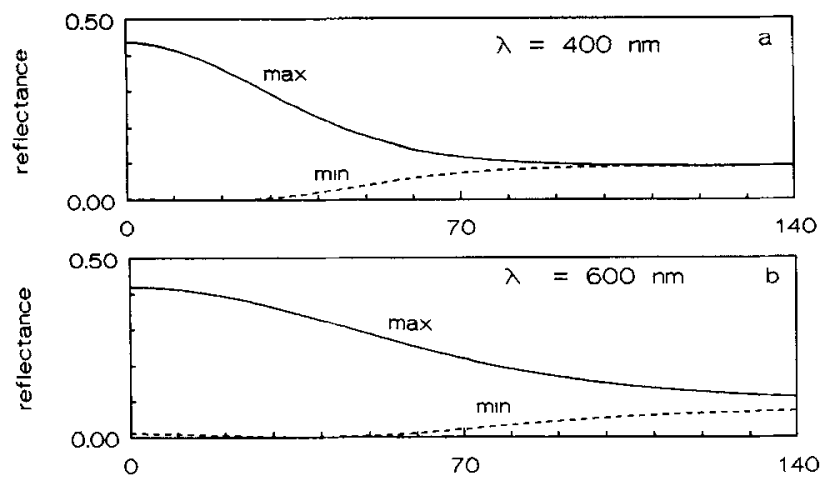

rms roughness $(\mathrm{nm})$

Fig. 5. The maximum and minimum reflectance vs. the rms roughness.

the same rate as the $\mathrm{W}$ on the $\mathrm{Si}$ surface, then we can calculate that the onset of selectivity loss started when the $\mathrm{W}$ thickness was about $400 \mathrm{~nm}$.

We calculated the effect of selectivity loss on the reflectance with the estimates derived from Fig. 6 and an apparent initial roughness of $50 \mathrm{~nm}$. The selectivity loss can be simulated by allowing a $\mathrm{W} / \mathrm{SiO}_{2} / \mathrm{Si}$ stack to grow in vertical and lateral dimension at the expense of the $\mathrm{SiO}_{2} / \mathrm{Si}$ stack. A Gaussian distribution of the roughness was assumed for both the $\mathrm{W}$ on $\mathrm{Si}$ and the $\mathrm{W}$ on $\mathrm{SiO}_{2}$.

The results are presented in Fig. $7 \mathrm{a}$ and $\mathrm{b}$ for $\lambda=400$ and $600 \mathrm{~nm}$, respectively. This agrees with the experiments.

In situ $\mathrm{NF}_{3}$ plasma cleaning.-The reactor is cleaned in an afterglow $\mathrm{NF}_{3}$ plasma after each deposition. The availability of such an afterglow plasma also allows us to clean the wafer in situ in the reactor just prior to the start of the deposition sequence. It was found that such a treatment strongly extends the period of selective deposition. A disadvantage is that this plasma not only etches the oxide but also and even stronger the $\mathrm{Si}$. The $\mathrm{Si}$ etching is very irregular as can be seen in Fig. 8. The $\mathrm{NF}_{3}$ plasma treatment is still under study and more details will be presented later.

The reflectance-time relation during the deposition via reaction [8] only was measured, and the results are presented in Fig. 9.

The SEM photograph (Fig. $10 \mathrm{a}$ and b) corresponding with the result presented in Fig. 9 was taken at an angle of $30^{\circ}$ with respect to the normal. From Fig. 10 the following conclusions can be drawn:

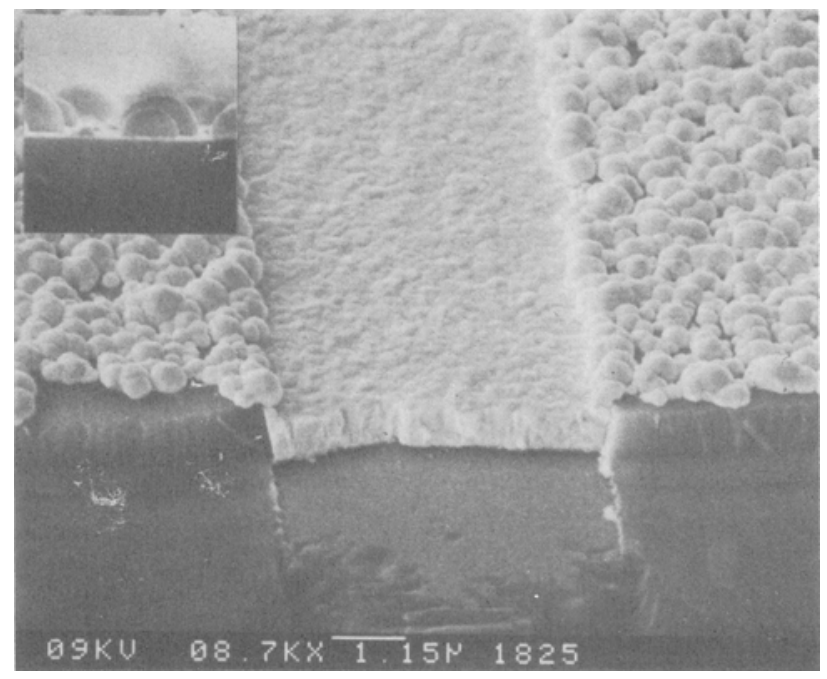

Fig. 6. Cross-sectional SEM photograph taken from the experiment presented in Fig. $4 \mathrm{a}$. The specimen was tilted $30^{\circ}$ with respect to the normal. The insertion shows the hemispheres on the oxide from a specimen which was stopped in an earlier stage at $0^{\circ}$ tilt with respect to the normal.
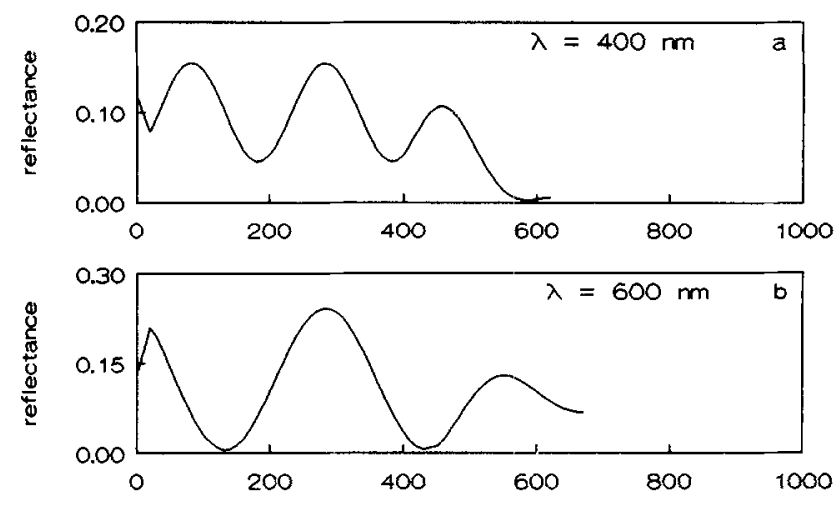

$W$ thickness (mm)

Fig. 7. Simulation of the reflectance including selectivity loss and an initial roughness of $50 \mathrm{~nm}$.

1. The $\mathrm{W}$ thickness is $2.1 \mu \mathrm{m}$ which is in agreement with the reflectance measurement provided that we assume that the small reflectance change during the first minute is not due to an induction period but due to a very small amplitude caused by a rough surface with $\mathrm{rms}>100 \mathrm{~nm}$. From Fig. 8 we may indeed conclude that the roughness after the $\mathrm{NF}_{3}$ clean is large.

2. The oxide thickness in this case was $700 \mathrm{~nm}$, in agreement with ellipsometer data.

3. The lateral overgrowth starts when the grating is completely filled. The lateral growth rate is equal to the vertical growth rate.

4. The amount of Si etched by the $\mathrm{NF}_{3}$ plasma is about $0.3 \mu \mathrm{m}$.

5. W hemispheres on the oxide have a typical radius of $0.6 \mu \mathrm{m}$, indicating that selectivity loss on the oxide started at a $W$ thickness of about $1.5 \mu \mathrm{m}$.

6 . The initial surface roughness caused by the $\mathrm{NF}_{3}$ plasma and visible in Fig. 10a where part of the $W$ was peeled off, is not replicated in the $W$.

From Fig. 9 it can be seen that the amplitude increases with thickness from nearly zero to a maximum and then decreases again, the minimum reflectance decreases continuously; also note that the distance between minima and maxima increases when the thickness of the $W$ is beyond $1 \mu \mathrm{m}$. The growth rate as calculated from Fig. 9 is presented in Fig. 11. The phenomenon of growth rate decay can be attributed to selectivity loss on the susceptor which was observed visually after about $2.5 \mathrm{~min}$. It is also the point where the lateral overgrowth starts. The surface concentration of reactants depends on the total reactant consuming area as was found earlier for the $\mathrm{H}_{2}$ chemistry $(19,20)$. The $\mathrm{SiH}_{4}$ surface concentration especially is of importance since the rate of reaction $[8]$ is linear proportional with the $\mathrm{SiH}_{4}$ concentration (5).

In order to understand at least qualitatively the shape of the reflectance characteristic in Fig. 9 we simulated the reflectance under the following assumptions:

1. The initial rms surface roughness due to the $\mathrm{NF}_{3}$ plasma treatment and due to other sources as mentioned earlier was assumed to be $100 \mathrm{~nm}$. This roughness is as-

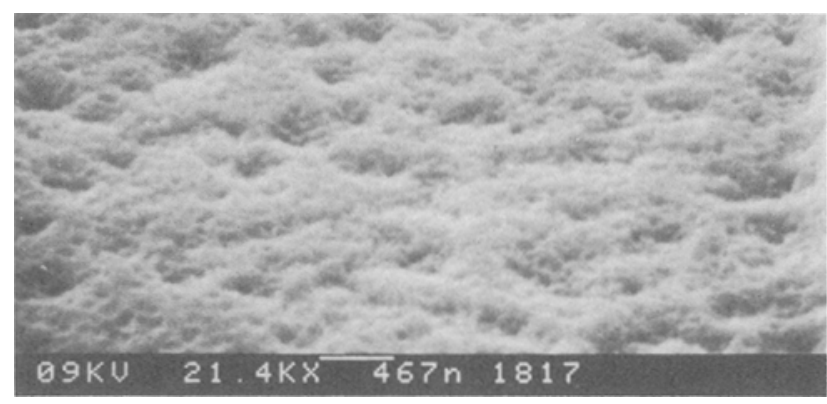

Fig. 8. SEM photograph of the Si surface after $15 \mathrm{~s}$ of an in situ $\mathrm{NF}_{3}$ plasma clean. The specimen is tilted $60^{\circ}$ with respect to the horizontal. 


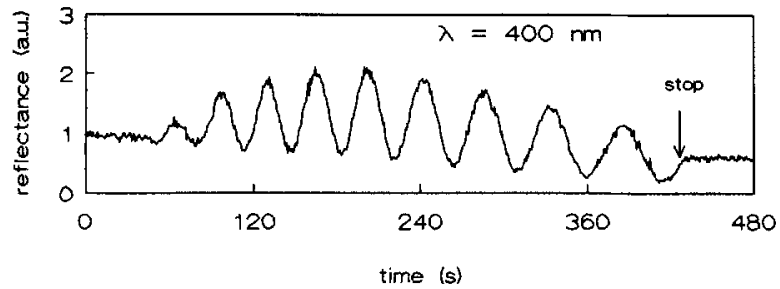

Fig. 9. Measured reflectance during $W$ deposition via reaction [8]. Pretreatment: $15 \mathrm{~s}$ of in situ $\mathrm{NF}_{3}$ plasma clean. 1 a.u. $=0.087$.

sumed to be build up of rectangular corrugations. The negative corrugations are assumed to become completely filled by the W when a W thickness of $1.0 \mu \mathrm{m}$ has been reached. No statistical variation of the lateral dimension of the corrugations is assumed.

2 . The roughness of the $W$ layer itself is assumed to be $3 \%$ of the W thickness.

3. The oxide thickness is $0.7 \mu \mathrm{m}$.

4. The amount of Si etched is assumed to be $0.3 \mu \mathrm{m}$.

5. Lateral overgrowth starts at a thickness of $1.0 \mu \mathrm{m}$; this is when the $\mathrm{W}$ thickness equals the $\mathrm{SiO}_{2}$ thickness plus the amount of etched Si.

6. No selectivity loss on the oxide is assumed.

The result of this simulation is presented in Fig. 12 and shows that the assumptions stated above fit the observation. An initial rms roughness of $120 \mathrm{~nm}$ would have fitted better and would have explained the low reflectance change during the first $60 \mathrm{~s}$. The goal of the calculation presented in Fig. 12 was however not to get quantitative agreement with the measurement but to understand the effect of smoothing and lateral overgrowth. Note that in all simulations the reflectance is plotted vs. thickness whereas the measured reflectance is plotted against time.
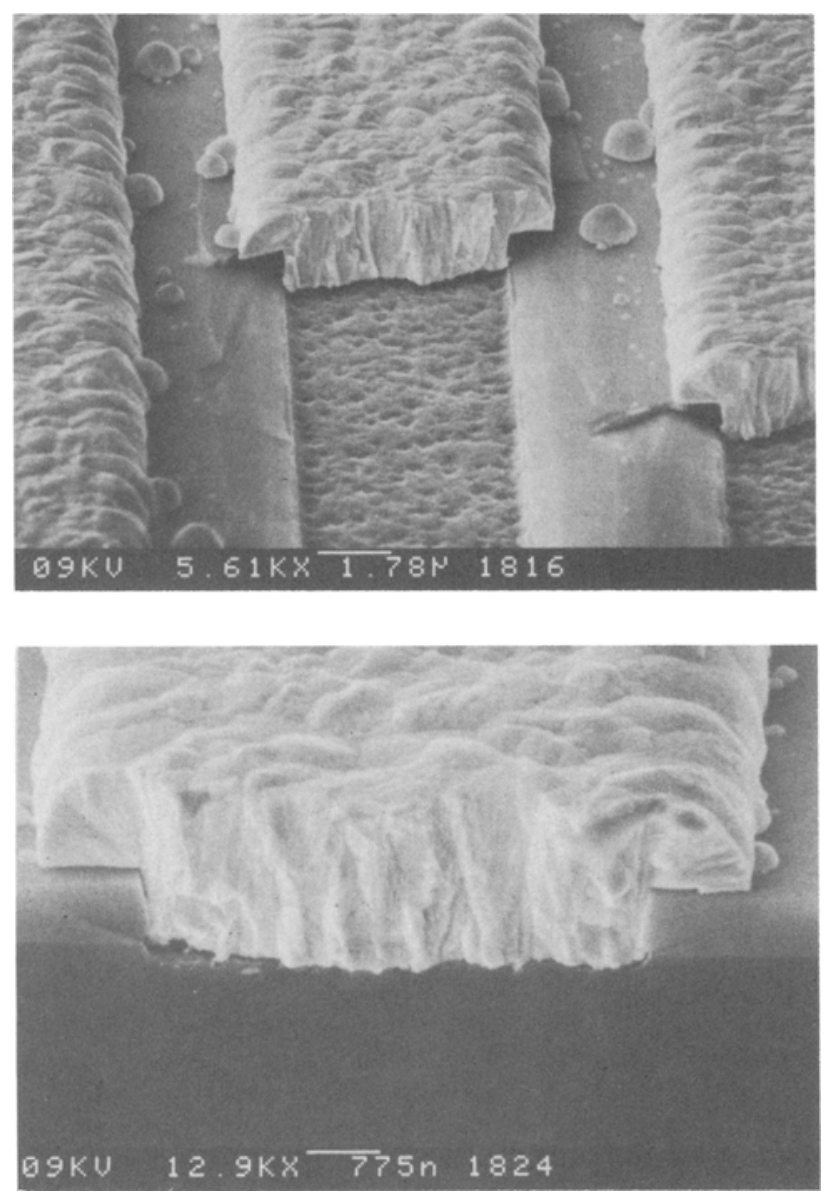

Fig. 10. Cross-sectional SEM photograph of the experiment presented in Fig. 9. ( $a$, top) $0^{\circ}$ tilt; (b, bottom) $30^{\circ}$ tilt with respect to the normal.

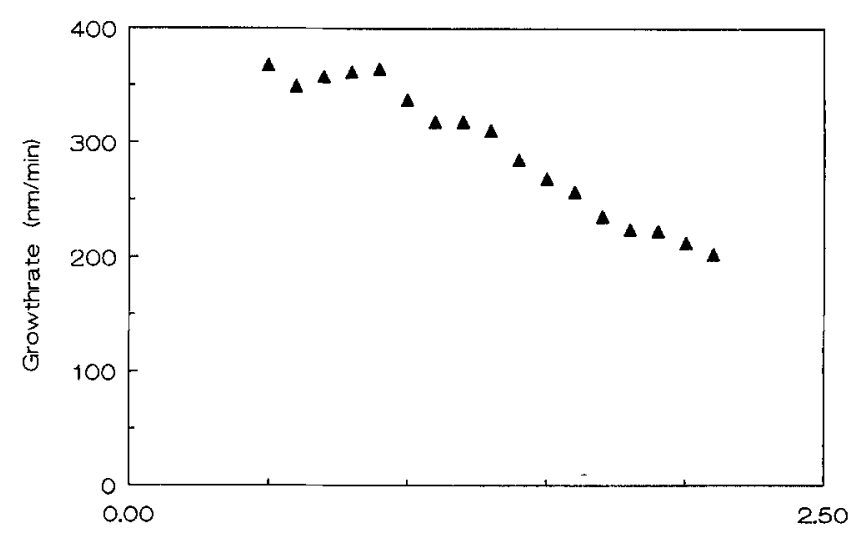

W thickness (um)

Fig. 11. Growth rate on a grating vs. $W$ thickness. $W F_{6}=40 \mathrm{sccm}$, $\mathrm{SiH}_{4}=30 \mathrm{sccm}, \mathrm{Ar}=1.5 \mathrm{sim}$, temp. $=300^{\circ} \mathrm{C}, p=0.2$ torr.

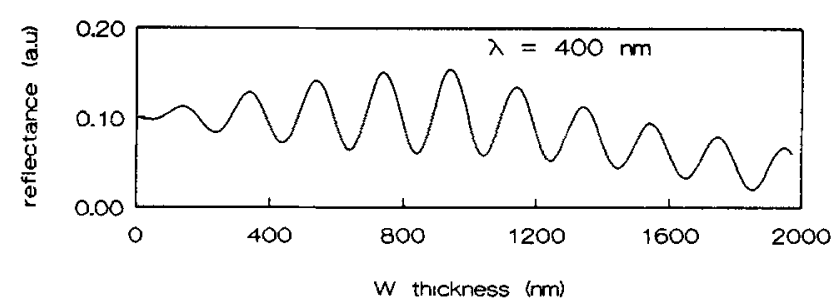

Fig. 12. Simulation of the reflectance during $W$ deposition via reaction [8], further assumptions can be found in the text.

\section{Conclusions}

The feasibility of the in situ growth rate measurement of selective $W$ has been demonstrated. The effect of surface roughness, selectivity, and later overgrowth on the reflectance is understood. A decreasing growth rate during the deposition process was measured, which may be related to an increasing surface area where deposition occurs. The increasing surface area may be related to lateral overgrowth and selectivity loss.

The method can also be applied for other selective deposition schemes.

\section{Acknowledgments}

This work forms part of the "Innovatief Onderzoeks Programma IC technologie" (Innovating Research Program for IC Technology) and was made possible by the financial support from the Netherlands Ministry of Economic Affairs.

Manuscript submitted June 28, 1990; revised manuscript received Oct. $10,1990$.

University of Twente assisted in meeting the publication costs of this article.

\section{REFERENCES}

1. G. F. Mendes, L. Cescato, and J. Frejlich, Appl. Optics, 23, 571 (1984).

2. G. F. Mendes, L. Cescato, J. Frejlich, E. S. Braga, and A. P. Mammana, This Journal, 132, 190 (1985).

3. P. A. Heimann, ibid., 132, 2003 (1985).

4. E. K. Broadbent and C. L. Ramiller, ibid., 131, 1427 (1984).

5. R. F. Foster, S. Tseng, and L. Lane, in "Proceedings of the 1987 Workshop on Tungsten and Other Refractory Metals for VLSI Applications III," V. A. Wells, Editor, p. 69, MRS, Pittsburgh, PA (1988).

6. T. Moriya, S. Shima, Y. Hazuki, M. Chiba, and M. Kashiwagi, IEDM T'ech. Dig., CH-1973-7/83, 550 (1983).

7. E. K. Broadbent and C. L. Ramiller, Abstract 420 , p. 657, The Electrochemical Society Extended Abstracts, Vol. 83-1, San Francisco, CA, May 8-13, 1983.

8. S. Swrihun, K. C. Saraswat, and R. M. Swanson, IEEE Electron Device Lett., EDL-5, 209 (1984).

9. A. Vasicek, "Optics of Thin Films," North Holland, Amsterdam (1960). 
10. C. van den Laan and H. J. Frankena, Appl. Optics, 17, 538 (1978).

11. T. I. Kamins, D. R. Bradbury, T. R. Cass, S. S. Laderman, and G. A. Reid, This Journal, 133, 2555 (1986).

12. J. Holleman, A. Hasper, and J. Middelhoek, in "Chemical Vapor Deposition of Refractory Metals and Ceramics," T. M. Besman and B. M. Gallois, Editors, p. 107, MRS Symposium Series, MRS, Pittsburgh, PA (1989).

13. J. O. Porteous, J. Opt. Soc. Am., 53, 1394 (1963).

14. P. Beckmann and A. Spizzichino, "The Scattering of Electromagnetic Waves from Rough Surfaces," Pergamon Press, Oxford (1963).

15. M. L. Green and R. A. Levy, This Journal, 132, 1243 (1985).
16. J. Holleman, A. Hasper, and J. Middelhoek, This Journal, 138, 783 (1991).

17. "Handbook of Optical Constants of Solids," E. D. Palik, Editor, Academic Press, Inc., New York (1985).

18. T. Sato, Jpn. J. Appl. Phys., 6, 339 (1967).

19. C. R. Kleijn, A. Hasper, C. J. Hoogendoorn, J. Holleman, and J. Middelhoek, in "Proceedings of the 1989 Workshop on Tungsten and Other Advanced Metals for VLSI, ULSI Application V," S. S. Wong and S. Furukawa, Editors, p. 109, MRS, Pittsburgh, PA (1990).

20. C. R. Kleijn, C. J. Hoogendoorn, A. Hasper, J. Holleman, and J. Middelhoek, This Journal, 138, 509 (1991).

\title{
Composition, Growth Mechanism, and Oxidation of Anodic Fluoride Films on $\mathrm{Hg}_{1-x} \mathrm{Cd}_{x} \mathrm{Te}(x \sim 0.2)$
}

\author{
Eliezer Weiss ${ }^{1}$ and C. R. Helms * \\ Department of Electrical Engineering, Stanford University, Stanford, California 94305
}

\begin{abstract}
Fluoridic films produced by the anodization of $\mathrm{Hg}_{1-x} \mathrm{Cd}_{x} \mathrm{Te}(x \sim 0.22)$ surfaces in nonaqueous solutions were characterized by Auger electron spectroscopy, x-ray photoelectron spectroscopy, and electron microprobe analysis. The films consist of three distinct regions. A thick uniform region, containing the fluorides of cadmium, mercury, and tellurium, and $\mathrm{HgTe}$, is covered by a thin $\mathrm{CdF}_{2}$ layer. The third region-the film-substrate interface-poor in mercury, consists mainly of $\mathrm{CdF}_{2}$ and $\mathrm{TeF}_{4}$. Using ultra-thin Pd marker layers and growth from baths saturated in $\mathrm{CdF}_{2}$ it is shown that these anodic films are grown by two mechanisms: the dominant one occurs by motion of the film-substrate interface into the semiconductor, consuming the original surface. There is, however, some growth at the film-electrolyte interface which forms the thin $\mathrm{CdF}_{2}$ layer on top of the structure. This second mechanism has a crucial effect on the stability of the film against oxidation. The $\mathrm{CdF}_{2}$ rich region acts as a diffusion barrier for the in-diffusion of oxidizing species. Tellurium ions, on the other hand, diffuse to the outer surface to be oxidized there.
\end{abstract}

Recently Weiss and Mainzer reported the passivation of $\mathrm{Hg}_{1-x} \mathrm{Cd}_{x} \mathrm{Te}$ surfaces by growing anodic layers from fluoridic solutions $(1,2)$. The use of anodic fluoridization or fluoro-oxidation is advantageous because the resulting interfaces are relatively thermally stable. Moreover, this method also reduces the surface recombination velocity and enables the adjustment of the amount of band bending at the surface of $\mathrm{Hg}_{1-x} \mathrm{Cd}_{x} \mathrm{Te}$ infrared detectors.

The films were grown anodically in either aqueous or nonaqueous solutions of $\mathrm{KF}$ or $\mathrm{KF}+\mathrm{KOH}$ in ethylene gly$\mathrm{col}(\mathrm{EG})$. An anodic fluoride, free of oxygen, is grown from nonaqueous solutions. Even low hydroxyl ion concentration in an aqueous bath causes the growth of an anodic fluoro-oxide, consisting of an oxide and small amounts of fluorides (1). An analysis, using a low-energy proton induced nuclear reaction, which is very sensitive to fluorine atoms, showed that in both cases fluorine is accumulated at the film-semiconductor interface and on the film surface (2). It was stated that the anodic fluoride is composed of cadmium fluoride in a matrix of tellurium, cadmium, and mercury. Voltage $v s$. time $(V-t)$ curves recorded during the anodic growth of fluorides and fluoro-oxides are similar to those obtained in the growth of anodic oxides on $\mathrm{Hg}_{1-x} \mathrm{Cd}_{x} \mathrm{Te}$, in contrast to those obtained for anodic sulfides $(1,2)$. It is believed, therefore, that the anodic fluoride films are grown by a mechanism similar to that of the anodic oxide.

The objective of the present work is to further characterize the anodic fluoride films on $\mathrm{Hg}_{1_{x}} \mathrm{Cd}_{x} \mathrm{Te}$ in terms of their composition, growth mechanism, and post-growth oxidation. We have concentrated in this work only on the anodic growth in nonaqueous solutions of KF. That is, on the anodic fluoridization as opposed to the fluoro-oxidation process. Our approach has emphasized the use of

* Electrochemical Society Active Member

1 Permanent address: SCD-Semi-Conductor Devices, D.N. Misgav 20179, Israel. several techniques in order to answer the various questions concerning these films. Auger depth profiling was employed to monitor the changes of the composition of the fluoride films. The chemical nature of the films was determined from studying the chemical shifts and line shape changes in both the Auger electron spectroscopy (AES) and the x-ray photoelectron spectroscopy (XPS) analyses. An electron microprobe was used to quantitatively analyze the films.

We found the anodic film consists of three distinct regions: cadmium fluoride-rich layers accumulate at both interfaces of a thick uniform region, which consists of the fluorides of all three constituents of the substrate embedded in mercury telluride. The whole structure is covered with a thin layer of $\mathrm{TeO}_{2}$, due to oxidation by water vapor. The film is grown by two mechanisms: the dominant one occurs by the motion of the film-substrate interface into the semiconductor, consuming the original surface. There is, however, some growth at the film-electrolyte interface which forms the thin $\mathrm{CdF}_{2}$ layer on top of the structure. It turns out that this second mechanism has a crucial effect on the stability of the film against oxidation. Films which were grown under conditions such that the top $\mathrm{CdF}_{2}$-rich region is absent and films in which this top layer was carefully etched off are oxidized rapidly upon exposure to room ambient.

\section{Experimental Details}

The $\mathrm{Hg}_{1-x} \mathrm{Cd}_{x} \mathrm{Te}$ wafers to be anodically fluoridized were grown by solid-state recrystallization. They were singlecrystalline $n$-type samples of random crystallographic orientation with $x \sim 0.22$. Before anodization the samples were degreased in trichloroethane, acetone, and 2-propanol and oxide stripped in $15 \%$ volume (v/o) lactic acid. Roughly $8000 \AA$ of the $\mathrm{Hg}_{1-x} \mathrm{Cd}_{x} \mathrm{Te}$ and previous anodic films were etched, using $0.12 \mathrm{v} / \mathrm{o}$ bromine-methanol solution, after which the samples were thoroughly rinsed in 